\title{
Less is more, regularly: metronomic dosing of cytotoxic drugs can target tumor angiogenesis in mice
}

\author{
Douglas Hanahan,,$^{1,2}$ Gabriele Bergers, ${ }^{1,2}$ and Emily Bergsland ${ }^{2,3}$ \\ ${ }^{1}$ Department of Biochemistry and Biophysics, \\ ${ }^{2}$ Hormone Research Institute, and \\ ${ }^{3}$ Department of Medicine, University of California San Francisco, San Francisco, California, USA
}

Address correspondence to: Douglas Hanahan, Departments of Biochemistry and Biophysics, and Hormone Research Institute, University of California San Francisco, San Francisco, California 94143-0534, USA. Phone: (415) 476-9209; Fax: (415) 731-3612;

E-mail: dh@biochem.ucsf.edu.

Chemotherapeutic drugs, long the mainstay of cancer treatment, cause DNA damage and disrupt DNA replication in proliferating cells. Drug regimens have been designed to kill as many tumor cells as possible by treating with "maximum tolerated doses" (MTDs) of these cytotoxic agents. Side effects such as neurotoxicity and damage to proliferating cells in healthy tissues pose serious constraints on the use of chemotherapy. In an effort to balance toxicity with efficacy, a conventional dosing schedule calls for episodic application of a cytotoxic drug at or near the MTD, followed by periods of rest to allow normal tissues to recover. Many such chemotherapy regimens are initially efficacious, resulting in tumor regression or stabilization and prolonged survival. In rare cases, cures are achieved. In general, however, responses are short-lived, with relapses often marked by aggressive cancers that are resistant to the cytotoxic drug. Furthermore, the standard MTD regimen as a rule seriously impairs quality of life.

The harsh side effects and the ultimate failures of most chemotherapies have fueled broad investigation of alternatives, including drugs that target not the transformed tumor cells themselves, but rather a genetically stable constituent cell type of tumors, the endothelial cells that form blood vessels. Angiogenesis, the process by which new blood vessels are formed, is a hallmark capability of cancer (1); a compelling body of evidence argues that tumor growth depends on the vasculature, and, in particular, on continuing angiogenesis $(2,3)$. More than two dozen new drugs that are in or soon to enter clinical trials appear to interfere with tumor angiogenesis $(3,4)$; there is considerable anticipation about their benefits in treating cancer. Now, two studies suggest a potentially complementary strategy of rescheduling the administration of classical cytotoxic drugs in order to target tumor endothelial cells.

It is well established that tumorassociated endothelial cells proliferate during chronic angiogenesis in tumors, albeit at lower frequencies than the tumor cells themselves. Apparently because of their lower rate of cell division, replication of these endothelial cells is only weakly disrupted by the episodic regimens of standard chemotherapeutic protocols. In these two new studies, however, cytotoxic drugs were administered routinely, to target the slowly proliferating tumor endothelial cells and abrogate their apparent capability to repair and recover during the usual rest periods. Both groups worked with mice bearing subcutaneous tumors, and each presents data suggesting that "metronomic" dosing regimens-either continuous infusion or frequent administration without extended rest periods-could have real value in the clinic. Both also demonstrated combinatorial effects of such altered cytotoxic drug regimens with newer, more specific angiogenesis inhibitors.

\section{Metronomic drug delivery in immunodeficient mice}

Klement and colleagues, in this issue of the JCI, tested two agents on tumors arising from human neuroblastoma cell lines, inoculated into immunodeficient mice (5). The first agent, the mitosis-blocking cytotoxic drug vinblastine, killed cultured endothelial cells at doses considerably below those required to affect the drug-sensitive neuroblastoma cells directly. Comparable doses, well below the MTD, impaired but did not abolish tumor growth in mice. Klement et al. (5) also explored the use of the mAb DC-101 (6), which disrupts the function of VEGF-R2/flk-1/KDR, one of two receptors for VEGF. VEGF signaling can induce endothelial cell proliferation and angiogenesis; in addition, accumulating data indicate that VEGF is a survival factor that protects endothelial cells from apoptosis (7). Treatment with DC101 impaired tumor growth, more so than vinblastine alone, but each agent alone produced only a period of "stable disease," after which lethal tumor growth resumed.

Remarkably, regular administration of the two drugs in combination produced regression of tumors, with no recurrence during 180 days of treatment. Histopathology revealed widespread apoptosis in tumors from all three treatment groups, notably in endothelial cells; cell death was most pronounced in the combination trial. The case for both agents having antiangiogenic activity was bolstered with an angiogenesis bioassay involving ingrowth of capillaries into subcutaneous matrix plugs containing an angiogenic growth factor: vinblastine and DC101 alone, and in combination, inhibited angiogenesis. Another group has independently documented the antiangiogenic effects of low-dose vinblastine (8). In recent unpublished work, the combination of metronomic, low-dose chemotherapy and antiVEGFR2 has been found to stabilize or regress tumors derived from several human breast cancer lines resistant to the cognate cytotoxic drug, supporting the generality of the strategy (G. Klement and R. Kerbel, personal communication). 


\section{Renewed promise for discarded drugs}

In a parallel study, Browder et al. (9) grew cyclophosphamide-resistant tumors (Lewis lung carcinoma and EMT-6 breast carcinoma) in immunocompetent mice to focus more specifically on the effects of a cytotoxic drug on tumor endothelium under different dosing schedule. Cyclophosphamide treatment in a conventional MTD regimen only modestly delayed growth of both tumor types in mice. In contrast, when cyclophosphamide was instead supplied regularly (once every 6 days), tumor growth was significantly impaired, although the tumors eventually prevailed. Provocatively, the relapse of drug-resistant Lewis Lung tumors could be prevented by a combination therapy, this time involving similar metronomic dosing with the experimental angiogenesis inhibitor TNP470. TNP-470 had previously been shown to impair but not regress subcutaneous tumor growth in mice (10) and to enhance high-dose episodic chemotherapy (11). In combination, cyclophosphamide and TNP-470 eradicated aggressive drug-resistant tumors in 32/38 tumor-bearing mice. Other trials assessed drug-sensitive Lewis Lung carcinomas and L1210 leukemias, both of which could be regressed without relapse using metronomic dosing of cyclophosphamide alone. The dose of cyclophosphamide used in this study was relatively high and resulted in significant toxicity; one wonders whether lower, nontoxic levels of cyclophosphamide would have sufficed, in combination with TNP-470, to induce the regression of these tumors. As predicted, metronomic cytotoxic dosing elicited repeated waves of apoptosis of tumor endothelial cells. Using an angiogenesis bioassay in normal mice, Browder et al. (9) confirmed that metronomic dosing of cyclophosphamide, as well as of a number of other cytotoxic drugs (including 5 -fluorouracil [5-FU]), was antiangiogenic.
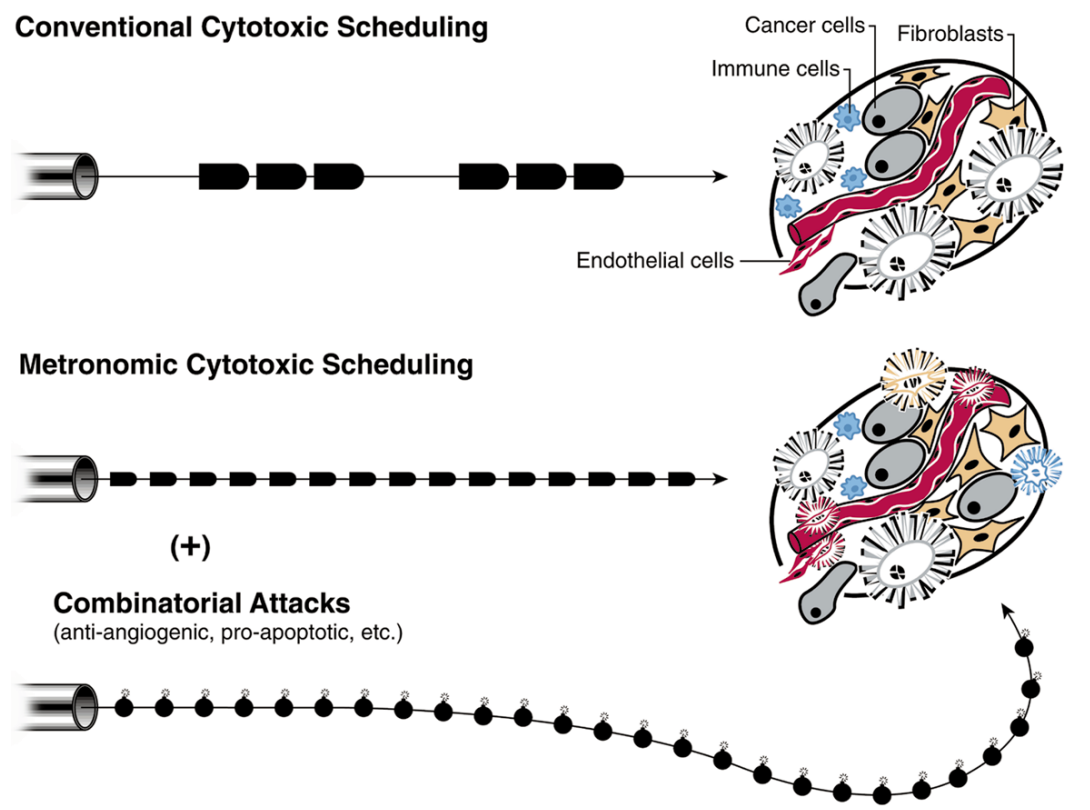

Figure 1

The changing logic of chemotherapy: metronomic dosing at lower levels, in combination with other agents. The conventional logic of chemotherapy has been to treat cancers with closely spaced bolus infusions of drugs at or near the MTD, followed by substantial rest periods. The typical results were transitory improvements in tumor burden and life-span extension accompanied by disturbing side effects and eventual relapse. The new logic of chemotherapy involves dosing at constant intervals without rest periods (metronomic scheduling), the use of lower doses to minimize toxic side effects and eliminate the obligatory rest periods, and combination with other drugs targeting distinct aspects of a cancer's functionality. The metronomic and combinatorial dosing strategies can kill tumor endothelial cells as well as overt cancer cells and, perhaps, other cellular constituents of a tumor, offering the prospect for genuine efficacy. The bullets refer to doses of chemotherapy, which can be large and episodic (upper scheme) or smaller and metronomic (lower); the bombs indicate other anti-cancer agents providing additive or synergistic benefits.

Collectively, these two studies $(5,9)$ clearly establish that metronomic regimens of cytotoxic drugs can be antiangiogenic, repositioning cytotoxic therapies as bi- or multifunctional against distinct heterotypic cell types in tumors (Figure 1). [The concept of antiangiogenic dosing was originated by Browder et al. (9), as noted by Klement et al. (5).] Both studies further demonstrated the value of combining modified chemotherapeutic regimens with experimental angiogenesis inhibitors. While the data presented are compelling, direct extrapolation to the clinical setting presents several immediate challenges related to the choice of drug, dose, and schedule for maximum antiangiogenic activity. In de-emphasizing the tumor cell as a target, this strategy requires a fundamental change in our approach to therapy, one that potentially includes retreatment of refractory cancers with agents that have previously failed, or the use of agents traditionally deemed inactive or ineffective in a particular cancer type. Second, identification of a MTD by standard toxicity criteria is relatively straightforward, whereas selecting the optimum antiangiogenic dose that is nontoxic yet efficacious may be difficult; surrogate markers of response and/or accurate preclinical models will be important. Novel imaging modalities designed to monitor angiogenesis may prove instrumental in this regard.

\section{Prospects for clinical application}

To put these results in perspective, it is important to note that we already have some experience with continuous or metronomic dosing in the clinical setting. Dozens of chemotherapeutic agents have been delivered by continuous infusion (12), although the interpretation of these studies is hampered by the lack of a standard definition of continuous infusion (ranging, in different studies, from 24 hours to several months) and the paucity of randomized clinical trials comparing bolus and continuous infusion. 5-FU stands out as the exception. Potentially consistent with Browder's observation (9) that metronomic dosing enhances the antiangiogenic activity of 5-FU, a recent meta-analysis of several randomized studies involving patients with metastatic colon cancer demonstrated a higher response rate with continuous-infusion therapy ( $22 \%$ vs. $14 \%$ ); the impact on survival, however, was trivial (13), and 
this regimen has not become standard practice. Additional clinical trials exploring chronic low-dose or continuous-infusion chemotherapy have met with limited success (14-17).

Although these data are sobering in the context of the current animal studies, it is important to note that continuous or chronic chemotherapy administration in the clinical setting has nearly always been undertaken using doses at or near the MTD, resulting in toxicity and requisite breaks from therapy. Hence, the value of chemotherapies administered at low doses on antiangiogenic schedules remains to be fully assessed. There are, meanwhile, encouraging anecdotal results; for example, from a study in which drugresistant patients with breast cancer were placed on a low-dose metronomic schedule involving the same cytotoxic drug (18). If long-term administration is to be achieved in practice, future studies should be aimed at identifying the optimal antiangiogenic agents, doses, and schedules, with special consideration to patient convenience, as well as toxicity and efficacy.

Despite these complimentary reports $(5,9)$ on the eradication of subcutaneous tumors in mice, it may be unrealistic to expect such dramatic results in humans. In particular, metronomic dosing with cytotoxic drugs, while demonstrably antiangiogenic, seem unlikely to prove efficacious in general as single agents. Nevertheless, we believe that metronomic delivery of lowered doses of cytotoxic drugs could be devised to minimize often devastating side effects of chemotherapy, while targeting endothelial and tumor cells. True efficacy may come only with combinatorial therapies, wherein novel cytotoxic dosing schedules are used in conjunction with other drugs or radiation. Possible combinations include other approved drugs, such as cox-2 inhibitors (19), thalidomide $(20)$, or IFN- $\alpha / \beta(3,21)$, as well as experimental drugs such as VEGF/ VEGF-receptor inhibitors, other angiogenesis inhibitors (e.g., TNP-470), proapoptotic drugs (22), or biotherapeutic agents such as oncolytic viruses (ref. 23; also see other articles in the current JCI Perspective series on cancer biotherapy). The possibilities raised by these studies are provocative and deserve further preclinical and clinical investigation.

\section{Acknowledgments}

We thank Terry Schoop of Biomed Arts, San Francisco, for preparation of the figure.

1. Hanahan, D., and Weinberg, R.A. 2000. The hallmarks of cancer. Cell. 100:57-70.

2. Hanahan, D., and Folkman, J. 1996. Patterns and emerging mechanisms of the angiogenic switch during tumorigenesis. Cell. 86:353-364.

3. Folkman, J. 2000. Tumor angiogenesis. In Cancer medicine. Holland et al., editors. B.C. Decker Inc. Hamilton, Ontario, Canada. In press.

4. Cancer trials: news and features. Angiogenesis inhibitors in clinical trials. http://cancertrials.nci. nih.gov/news/angio/table.html.

5. Klement, G., et al. 2000. Continuous low-dose therapy with vinblastine and VEGF receptor- 2 antibody induces sustained tumor regression without overt toxicity. J. Clin. Invest. 105:R15-R24.

6. Witte, L., et al. 1998. Monoclonal antibodies targeting the VEGF receptor-2 (Flk1/KDR) as an antiangiogenic therapeutic strategy. Cancer Metastasis Rev. 17:155-161.

7. Benjamin, L.E., Golijanin, D., Itin, A., Pode, D., and Keshet, E. 1999. Selective ablation of immature blood vessels in established human tumors follows vascular endothelial growth factor withdrawal. $J$ Clin. Invest. 103:159-165.

8. Vacca, A., et al. 1999. Antiangiogenesis is produced by nontoxic doses of vinblastine. Blood. 94:4143-4155.

9. Browder, T., et al. 2000. Antiangiogenic scheduling of chemotherapy improves efficacy against experimental drug-resistant cancer. Cancer Res. 60:1878-1886.

10. Ingber, D., et al. 1990. Synthetic analogues of fumagillin that inhibit angiogenesis and suppress tumor growth. Nature. 348:555-557.

11. Teicher, B.A., et al. 1994. Potentiation of cytotoxic cancer therapies by TNP-470 alone and with other anti-angiogenic agents. Int. J. Cancer. 57:920-925.

12. Lokich, J., and Anderson, N. 1997. Dose intensity for bolus versus infusion chemotherapy administration: review of the literature for 27 anti-neoplastic agents. Ann. Oncol. 8:15-25.

13. The Meta-analysis Group in Cancer. 1998. Efficacy of intravenous continuous infusion to fluorouracil compared with bolus administration in advanced colorectal cancer. J. Clin. Oncol. 16:301-308.

14. Blumenreich, M.S., et al. 1994. Inefficacy of lowdose continuous oral etoposide in non-small cell lung cancer. Am. J. Clin. Oncol. 17:163-165.

15. Burris, H. 1998. Weekly schedules of docetaxel. Semin. Oncol. 25(Suppl. 13):21-23.

16. Sorensen, P., Andersen, L.J., Hansen, O., and Bastholt, L. 1999. Long-term continuous 5-fluorouracil infusion in patients with advanced head and neck cancer. Acta Oncol. 38:1043-1045.

17. Regazzoni, S., Pesce, G., Marini, G., Cavalli, F., and Goldhirsch, A. 1996. Low-dose continuous intravenous infusion of 5-fluorouracil for metastatic breast cancer. Ann. Oncol. 7:807-813.

18. Rocca, A.M., et al. 1999. Low dose oral methotrexate (MTX) and cyclophosphamide (CTX) in metastatic breast cancer (MBC): antitumor activity and correlation with serum vascular endothelial growth factor (VEGF) levels. Proc. Am. Soc. Clin. Oncol. 18:121a. (Abstr.)

19. Masferrer, J.L., Koki, A., and Seibert, A. 1999. COX2 inhibitor. A new class of antiangiogenic agents. Ann. NY Acad. Sci. 889:84-86.

20. D’Amato, R.J., Loughnan, M.S., Flynn, E., and Folkman, J. 1994. Thalidomide is an inhibitor of angiogenesis. Proc. Natl. Acad. Sci. USA. 91:4082-4085.

21. Slaton, J.W., Perrotte, P., Inoue, K., Dinney, C.P.N., and Fidler, I.J. 1999. Interferon- $\alpha$-mediated downregulation of angiogenesis-related genes and therapy of bladder cancer are dependent on optimization of biological dose and schedule. Clin. Cancer Res. 5:2726-2734.

22. Ashkenazi, A., et al. 1999. Safety and antitumor activity of recombinant soluble Apo2 ligand. J. Clin. Invest. 104:155-162.

23. Heise, C., and Kirn, D.H. 2000. Replication-sensitive adenoviruses as oncolytic agents. J. Clin. Invest. 105:847-851. 